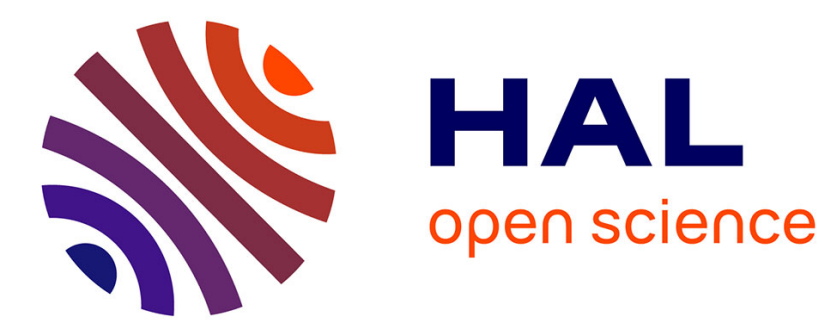

\title{
Analyse de réseaux en intelligence économique: éléments pour une approche méthodologique
}

Christian Marcon

\section{To cite this version:}

Christian Marcon. Analyse de réseaux en intelligence économique: éléments pour une approche méthodologique. Marketing et Communication, 2007, 4, pp.110-134. hal-01137816

\section{HAL Id: hal-01137816 https://hal.science/hal-01137816}

Submitted on 31 Mar 2015

HAL is a multi-disciplinary open access archive for the deposit and dissemination of scientific research documents, whether they are published or not. The documents may come from teaching and research institutions in France or abroad, or from public or private research centers.
L'archive ouverte pluridisciplinaire HAL, est destinée au dépôt et à la diffusion de documents scientifiques de niveau recherche, publiés ou non, émanant des établissements d'enseignement et de recherche français ou étrangers, des laboratoires publics ou privés. 


\title{
Analyse de réseaux en intelligence économique : éléments pour une approche méthodologique
}

\author{
Christian Marcon \\ Maître de conférences \\ Equipe Intelligence économique - CEREGE / Université de Poitiers
}

Depuis sa véritable reconnaissance en tant que champ professionnel en 1994 dans le cadre du rapport Henri martre, l'intelligence économique a fait l'objet de recherches nombreuses, tant de la part du monde académique que de celui des professionnels. Ces recherches ont labouré le terrain de plusieurs thématiques : exploration de la notion de guerre économique, interrogation sur les dispositifs de veille à mettre en oeuvre et en particulier sur les logiciels susceptibles de contribuer à de tels dispositifs, questionnement sur la rentabilité de l'intelligence économique et les conditions de sa mise en oeuvre dans des entreprises de dimensions extrêmement variables, benchmark des systèmes étrangers susceptibles de fournir des leçons pour une meilleure approche nationale...

De manière assez étonnante, la contribution des réseaux à l'intelligence économique est restée relativement peu étudiée. Si chacun semble s'accorder aujourd'hui à reconnaître l'importance de l'appui sur les réseaux, seuls quelques travaux ont commencé véritablement à en explorer les contours, les mécanismes, les modes d'activation...

Parmi ces travaux, quelques uns ont recommandé la mise en œuvre de dispositifs que nous qualifierons de discrètement réticulés. L'observatoire de l'environnement de Martinet et Ribaud [1989] résumait ainsi le réseau à un ensemble de correspondants " renseignement » opérationnels, relativement disponibles, connaissant beaucoup de monde dans l'entreprise, proches du terrain, ayant des qualités de veilleur et capables d'être les confidents des membres de leurs services pour tout ce qui concerne la veille ${ }^{1}$. L'organisation cellulaire proposée par Philippe Baumart [1990] articulait à son tour cellules opérationnelles et cellules de coordination, travail interne et appel à des prestataires extérieurs, éventuellement organisation matricielle pour une très grande entreprise à dimension internationale ${ }^{2}$. La place des réseaux, sous-jacente au dispositif, ne faisait pas l'objet d'une description développée. De même, l'organisation trinômiale de Pichot-Duclos [1994] semblait peu intégrer les réseaux ${ }^{3}$.

\footnotetext{
1 MARTINET Bruno, RIBAULT Jean-Michel, «La veille technologique concurrentielle et commerciale », Les éditions d'organisation, Paris, 1989, p. 247-248

2 BAUMART Philippe, "Stratégie et surveillance des environnements concurrentiels », Editions Masson, Paris, 1991, p. 143 et suiv.

3 PICHOT-DUCLOS Jean, "L'intelligence économique : pour un modèle français », Défense Nationale, janvier 1994, p. 73 à 85
} 
Plus récemment, d'autres approches ont suggéré des configurations plus fermement réticulées. II revient à Bernard Besson et Jean Claude Possin [1996], puis à François Jakobiak [1998] d'avoir explicitement développé la place des réseaux dans le dispositif d'intelligence économique. Pour les premiers, l'intelligence économique s'appuie sur la détection des réseaux de compétence et d'analyse internes, pilotés par un délégué général connecté directement à la direction ${ }^{4}$. Le modèle présente deux qualités : une structure légère et une volonté d'implication collective par les réseaux. François Jakobiak va plus loin en décrivant un dispositif d'intelligence économique qui s'organise autour de trois réseaux : un réseau d'observateurs professionnels de l'information documentaire, chargé de la recherche, de la collecte et de la diffusion des informations ; un réseau d'analyseurs - experts, chargé d'apporter de la valeur à l'information ; un réseau de décideurs ${ }^{5}$. Dans pratiquement tous les cas, ce sont les réseaux internes qui sont recherchés explicitement. L'appui sur les réseaux externes est peu formulé.

Désormais, les recommandations en matière d'organisation de l'intelligence économique incorporent toutes une dimension réseau forte, dont l'étendue, la forme, la dynamique, le rôle sont plus ou moins explicités. Le référentiel intelligence économique de l'AFDIE intègre par exemple le réseau parmi ses sept facteurs d'action: "le réseau est le nouveau mode d'organisation des entreprises qui vivent dans un monde de réseaux et qui sont elles-mêmes des réseaux. Ces réseaux doivent être identifiés. L'intelligence économique est, pour partie, la coordination et l'animation de ces réseaux. ${ }^{6}$ " Le référentiel complète cet exposé de principe par quelques recommandations générales pour lesquelles aucune précision méthodologique n'est donnée : description des réseaux existants dans l'entreprise, internes ou externes, professionnels ou non; amélioration du fonctionnement des réseaux, notamment par la centralisation de l'information et la facilitation de son accès ; création de réseaux ad hoc en fonction des besoins... Le référentiel de formation issu des travaux du groupe placé sous l'autorité d'Alain Juillet a lui-même émis quelques recommandations, assez timides, quant à l'étude de la dimension réseau dans les programmes d'intelligence économique. Mais l'ensemble reste très parcellaire.

Notre propos est donc d'avancer dans la recherche de repères méthodologiques pour la mise en oeuvre de réseaux au service d'une démarche d'intelligence économique. Pour ce faire, afin de sortir d'un discours de conviction et d'entrer dans un discours de démonstration, nous nous efforcerons d'abord de prendre appui sur des résultats de recherches qui éclairent le fonctionnement et le rôle des réseaux (1). Puis, dans un second temps, nous proposerons un schéma d'ensemble d'exploitation des réseaux dans le contexte de l'intelligence économique d'une entreprise et développerons sept recommandations méthodologiques.

\section{Repères pour l'analyse de réseaux en intelligence économique.}

Au-delà du discours de praticiens globalement convaincus, l'analyse des réseaux dans le domaine spécifique de l'intelligence économique n'a pas encore fait l'objet de recherches dédiées suffisantes pour aboutir à des modèles, des figures stylisées, des théories complètes qui permettraient une mise en oeuvre optimale - si tant est que cette ambition ait

\footnotetext{
${ }^{4}$ BESSON Bernard et POSSIN Jean Claude, "Du renseignement à l'intelligence économique », Editions Dunod, Paris, 1996, p. 93-99

5 JAKOBIAK François, "L'intelligence économique en pratique », Editions d'organisation, Paris, 1998, p. 290

${ }^{6}$ LEVET Jean Louis et al., Présentation du référentiel AFDIE en intelligence économique, Actes des $4^{\mathrm{e}}$ rencontres nationales d'intelligence économique, 8 novembre 2001, p. 30
} 
un sens. De sorte que, pour s'efforcer de cadrer de manière rigoureuse une méthodologie de l'emploi des réseaux au service de l'intelligence économique, il convient de procéder par emprunts successifs à des disciplines qui ont, elles, produit un matériau élaboré : la sociologie, les sciences de gestion et les sciences économiques ${ }^{7}$.

En puisant dans ces trois domaines, nous pouvons éclairer la question des réseaux en intelligence économique et ouvrir ainsi des pistes d'approche méthodologique.

\subsection{Analyse des structures relationnelles en réseau.}

\section{a. Inégalité des positions individuelles et stratégies}

Il est usuel de considérer que les premiers travaux marquants dans le domaine de l'analyse des réseaux sociaux sont ceux de Georg Simmel (1858-1918) qui, au début du XXe siècle, suggéra que les faits sociaux résultaient des interactions entre les individus plus que des individus eux-mêmes et que c'est à ce niveau là que la sociologie devait porter son regard ${ }^{8}$. Entre l'individualisme et le holisme méthodologique, cette approche ouvrait une troisième voie à l'analyse. Une voie qui s'est avérée féconde.

C'est en 1934 que Jacob Lévy Moreno (1889-1974), sociologue et psychiatre, fonda la sociométrie appelée aujourd'hui analyse structurale. Celle-ci a démontré que les positions des individus au sein des réseaux sont très inégales. Selon leur comportement relationnel, les individus sont plus ou moins centraux dans un réseau. De sorte que leur capacité d'action s'en trouve affectée. Ce phénomène de gravitation socio-affective, qui fait de certains des leaders et d'autres des isolés, des solitaires, ou des rejetés, est général. II s'ensuit que la capacité à agir en réseau et à se comporter en animateur de réseau est très variable d'un individu à l'autre.

L'évidente inégalité des positions personnelles dans les réseaux ne saurait être sans conséquences stratégiques, tant au niveau du dispositif réseau dans son ensemble que des attitudes personnelles.

Ronald Burt nomme structure sociale l'architecture des relations concrètes existant entre des personnes. La position de l'individu dans la structure sociale détermine son accès à un capital social, dans le sens que lui donne Pierre Bourdieu" : "l'agrégation des ressources

\footnotetext{
${ }^{7}$ Si I'on en croit l'analyse de Louis Lengrand et Isabelle Chatrie, " le concept de réseau a émergé dans les domaines de la sociologie et de la communication avant de s'étendre au domaine de l'économie et de la gestion. Ceci s'est produit relativement tardivement du fait de l'influence dominante des théories classiques et néoclassiques d'Adam Smith. Ces dernières reposaient sur l'individualisme économique, lui-même fondé sur la recherche du profit maximal par les individus et une concurrence féroce entre acteurs économiques. Cette situation où les acteurs ne défendaient généralement que leurs propres intérêts et où les opérateurs d'un même secteur se considéraient comme des concurrents ne laissaient aucune place au développement de réseaux de coopération interentreprises. " Louis Lengrand et Isabelle Chatrie, "Les réseaux d'entreprise et l'économie de la connaissance. Une étude de terrain mené en Europe et au Canada » une initiative de la direction générale d'entreprise de la commission européenne, novembre 1999, p.

${ }^{8}$ Sur ce point, et plus largement sur les origines de l'analyse des réseaux, voir l'article de Pierre Mercklé, "Les réseaux sociaux. Les origines de l'analyse des réseaux sociaux », CNED-ENS/Ish 2003-2004

9 BOURDIEU P. (1985), The forms of capital, in Richardson J.G. (éd.), Handbook of theory and research for the sociology of education, New-York, Greenwood, p. 241-258.
} 
effectives ou potentielles qui sont associées à la possession d'un réseau durable de relations plus ou moins institutionnalisées de connaissance mutuelle ou de reconnaissance ».

L'individu est donc tenté d'analyser ses «trous structuraux ${ }^{10}$ et de développer une stratégie personnelle pour améliorer son accès au capital social, stratégie dont rien ne permet d'affirmer qu'elle contribuera nécessairement au succès du réseau dans son ensemble. L'opportunisme est un risque avéré en ce domaine. On observe ainsi des stratégies variables chez les membres d'un réseau qui analysent leur position structurale comme trop périphérique. Certains développent une démarche visant à renforcer leur centralité : ils s'investissent dans des projets, acceptent des responsabilités, font en sorte de se faire apprécier par le collectif dans la perspective de devenir - osons cette expression triviale mais explicite - " calife à la place du calife ». D'autres, a contrario, cherchent à séduire une partie du réseau et à l'emporter avec eux dans une autre aventure, préférant susciter une scission. L'histoire des partis politiques, ancienne et récente, est riche d'exemples de ce choix stratégique. Gageons que celle des dispositifs d'intelligence économique, d'entreprise ou territoriaux, n'échappe pas à cette logique.

La constitution d'un réseau est donc sujette à analyses et stratégies complexes. Les auteurs divergent d'ailleurs sur les bénéfices automatiques ou non de la constitution d'un réseau. Pour Burt, déjà cité, si la constitution d'un réseau est souvent intentionnelle, elle permet de "recevoir des opportunités " naturellement. Au contraire, Nan Lin ${ }^{11}$, théoricien des ressources sociales, caractérise le capital social par " des ressources encastrées dans une structure sociale, dont l'accès est obtenu et/ou qui sont mobilisées lors d'actions intentionnelles ». Si l'on suit Burt, il suffit donc de développer son réseau pour bénéficier d'un effet réseau; si l'on en croît Lin, l'effet réseau résulte d'une action, d'une activation du réseau. II n'est pas question pour nous de soutenir l'une ou l'autre théorie, ni de chercher à les rapprocher. En revanche, nous suggérons que le doute doit prévaloir dans une logique d'intelligence économique: mieux vaut s'engager dans une approche méthodologique, volontariste pour bénéficier d'un effet réseau plutôt que d'escompter un bénéfice naturel.

\section{b. Les questions de force des liens et d'embeddedness}

L'une des approches les plus populaires des réseaux sociaux repose sur la distinction entre liens forts et de liens faibles, approche à laquelle Mark Granovetter a consacré un célèbre article paru dans la revue Sociological Theory ${ }^{12}$. Granovetter considère quatre critères qui contribuent au renforcement des liens au sein d'un réseau : la durée de la relation, l'intensité émotionnelle du vécu de cette relation, l'intimité entre les parties prenantes et enfin l'abondance des services rendus. Plus une relation sociale est longue, chargée de moments intenses (par exemple des difficultés professionnelles surmontées ensemble), a rapproché les individus et conduit à de nombreux échanges de services, et plus le lien sera considéré comme fort.

L'intérêt des liens "forts » est évidemment qu'ils soudent les individus, créent un esprit de corps, une solidarité profonde ${ }^{13}$. Appuyer sa stratégie-réseau sur la construction de liens forts, c'est aller à la recherche de bénéfices comparables à ceux que la stratégie d'entreprise

\footnotetext{
${ }^{10}$ Burt Ronald S. (1992), Structural Holes. The Social Structure of Competition, Cambridge (Mass.), Harvard University Press

11 LIN Nan, Les ressources sociales: une theorie du capital social, Revue Française de Sociologie, Vol. 36, No. 4, Analyses de reseaux et structures relationnelles (Oct. Dec., 1995), pp. 685-704

${ }^{12}$ GRANOVETTER Mark, The strengh of weak ties : a network theory revisited, Sociological Theory, volume 1 (1983), p. 201-233

${ }^{13}$ UCBASARAN, LOCKETT, WRIGHT \& WHESTHEAD (2003), Entrepreneurial Funders Teams: Factors Associated with Entry and Exit, Entrepreneurship Theory and Practice, pp. 107-128.
} 
dite de concentration, que des chercheurs comme M. Porter ont présenté depuis longtemps comme l'une des stratégies génériques fortes de l'entreprises. La limite de ce choix réside dans le repli sur soi qui peut frapper le réseau ainsi charpenté, au risque (et ce risque est essentiel en matière d'intelligence économique) de créer une cécité stratégique collective ${ }^{14}$. Le fameux syndrome NIH (Not Invented Here) n'a pas d'autre origine : rien de ce qui vient d'en dehors du groupe ne saurait valoir ce qui en provient. Difficile d'intégrer ce type de réseau ; difficile également d'en sortir. Difficile aussi, sans doute, de se mailler avec d'autres, dans le cadre d'une intelligence collective plus large.

Un fondement de l'approche de Granovetter, discuté néanmoins par B. Chollet dans sa thèse de doctorat ${ }^{15}$, est que les liens faibles sont plus utiles que les liens forts en ce qu'ils jettent des ponts vers des horizons culturels, cognitifs, sociaux et professionnels différents. En somme, ils ouvrent l'horizon stratégique de l'acteur, comme une stratégie de diversification d'entreprise lui ouvre les champs d'autres secteurs afin de multiplier les opportunités d'affaires ${ }^{16}$. Nous voyons là une opportunité en matière d'intelligence économique d'entreprise ou territoriale : celle de l'intelligence collective étendue, ouverte.

La dernière analyse qu'il faut ici évoquer est celle de l'encastrement - en anglais embeddedness - des activités économiques sur les liens sociaux. Les études menées dans le cadre de la sociologie économique, conduisent à la conclusion qu'une partie importante des réseaux générateurs d'activité économique ont pour origine des liens sociaux antérieurs. Bes et Grossetti $^{17}$, notent ainsi que dans près de $45 \%$ des cas, le développement de partenariats entre entreprises privées et laboratoires de recherche publique a pour origine une relation de type sociale entre les acteurs. Le travail de Nathalie Pinelli sur les réseaux corses confirme ce mécanisme dans cette culture bien spécifique ${ }^{18}$, mais d'autres exemples ne manquent pas, en particulier dans les fameux districts industriels italiens. Ce phénomène naturel serait particulièrement préoccupant s'il ne s'accompagnait d'un autre phénomène dit de découplage qui se traduit par la survie des réseaux à la disparition, ou tout au moins au retrait, de leurs fondateurs. Autrement dit, les liens sociaux sont, dans une proportion significative, source de création d'activités, lesquelles peuvent perdurer alors même que les liens sociaux initiaux disparaissent. Cela signifie donc que le support réseau crée les conditions de sa pérennité, soit par son institutionnalisation, soit par un mécanisme de translation sociale. Ce mécanisme permet la durabilité du réseau, nécessaire à l'apprentissage relationnel, lequel est le prélude à un véritable échange d'information si l'on suit la séquence analysée par Régis Debray ${ }^{19}$.

En somme, l'intérêt des travaux des sociologues évoqués brièvement dans ce premier point est de récuser quelques lieux communs de l'approche réseau en matière d'intelligence économique, par exemple l'idée que la constitution de pôles de compétitivités serait naturellement génératrice de synergie entre leurs membres, que tout homme d'entreprise est

\footnotetext{
${ }^{14}$ ENSLEY, M.D., PEARSON, A.W., \& AMASON, A.C. (2002). Understanding the Dynamics of New Venture Top Management Teams: Cohesion, Conflict and New Venture Performance, Journal of Business Venturing, 17(4), 365-386.

${ }^{15}$ CHOLLET Barthélémy, Le rôle du réseau personnel de l'ingénieur en $R$ \& $D$ : le cas du secteur des micro et nano technologies, thèse de doctorat, décembre 2005

${ }^{16}$ D'une certaine manière, les plateformes de networking en ligne ont choisi plutôt cette orientation, tandis que les applications de Network Relationship Management héritée des logiciels de CRM ont choisi la précédente

${ }^{17}$ GROSSETTI Michel et BES Marie-Pierre, Les effets de la proximité spatiale dans les relations entre organisations: encastrements et découplages, Communication pour les 3éme journées de la Proximité, 13 et 14 décembre 2001, Centre d'étude des rationalités et des savoirs - Université Toulouse-le-Mirail, $27 \mathrm{p}$.

${ }_{18}$ PINELLI Nathalie, Corse, l'île réseau(x), mémoire pour la validation d'un master Intelligence Economique et Communication Stratégique - Université de Poitiers 2007.

${ }^{19}$ DEBRAY Régis, Introduction à la médiologie, Presses Universitaires de France, Paris, 2000
} 
naturellement homme de réseau ou que tout acteur que l'on désigne pour s'inscrire dans un dispositif d'intelligence économique ou d'intelligence territoriale sera un homme de réseau...

Dans tous les cas - il faut nous en convaincre - pour constituer un réseau d'intelligence économique il faut vérifier les capacités personnelles des acteurs à s'inscrire dans un tel dispositif et, plus globalement, à agir en réseau car, ainsi que l'écrit $M$. Giraudel ${ }^{20}$, qui reste néanmoins prudent sur l'ampleur de son terrain de validation, "les recherches sur le contenu des liens, la structure, et les attributs des alter du réseau personnel ne sont réellement pertinentes qu'à partir du moment où elles intègrent une dimension relative à la capacité de l'individu à mobiliser son réseau personnel. " Ceci est sans doute affaire de méthode, et nous y viendrons. Mais voyons d'abord quels éléments d'analyse supplémentaires nous fournit l'analyse des coordinations inter firmes en réseaux..

\subsection{Approche de la coordination inter firmes en réseaux.}

L'une des questions que se pose la recherche en économie industrielle et en économie spatiale est celle de la coordination économique sur le marché, que Weinstein définit comme « les différentes procédures qui concourent à la compatibilité des comportements d'agents en interaction et permettent d'atteindre un certain degré d'efficience [. . . d dans l'obtention de résultats dépendant des actions de ces agents. La coordination se présente comme une succession d'actions individuelles en interaction; toute procédure de coordination ayant pour effet d'orienter et de contraindre les actions, donc de limiter le champ des actions possibles $^{21}$. "

Comment un marché s'organise-t-il et trouve-t-il son équilibre ? La concurrence assure-t-elle à elle seule, naturellement, cette organisation et cet équilibre ou bien d'autres facteurs entrent-ils en ligne de compte? Comment certaines entreprises tirent-elles mieux leur épingle du jeu ? Pourquoi certains territoires à forte concentration d'entreprises autour d'un secteur d'activité réussissent-ils mieux? Ces questions sont directement liées à notre réflexion sur la place des réseaux en intelligence économique. Depuis longtemps, des sujets comme la constitution d'alliances, le management de situations de coopétition - en particulier dans le contexte de la recherche, la coordination (afin de les préserver) de secteurs considérés comme constitutifs du patrimoine économique national, la guerre économique entre secteurs nationaux concurrents ou au sein d'un même secteur sont au cœur des préoccupations des professionnels et de l'Etat. Bernard Carayon préconisait ainsi dans son rapport que "sur la base d'un cahier des charges commun élaboré par l'instance politicoadministrative définie par le gouvernement, chaque ministère établi[sse] dans son champ de compétence, de manière prospective et après une large concertation avec ses partenaires naturels, la liste des organisations internationales, des secteurs d'activités, des technologies et des savoir-faire, des entreprises, des territoires-clefs pour le rayonnement, le développement économique de la France et le maintien de sa souveraineté et de l'emploi ${ }^{22}$ ».

Dans notre premier point, nous avons exploré l'une de ces approches, qui voit dans la coordination relationnelle une explication déterminante de la coordination inter firmes. D'autres sont d'un intérêt limité dans le contexte de cet article, telles la coordination par la

\footnotetext{
${ }^{20}$ GERAUDEL Mickaël, Les retombées du réseau personnel du dirigeant d'entreprise : la personnalité a-t-elle un rôle ? Actes de la $16^{\mathrm{e}}$ Conférence internationale du management stratégique, Montréal, juin 2007, p. 14

${ }^{21}$ WEINSTEIN Olivier, Modèles d'innovation et modes de coordination inter et intra-entreprises, in Palloix C., Rizopoulos Y. (Eds.), Firmes et économie industrielle, Paris, 1997, L'Harmattan, p. 390.

${ }^{22}$ CARAYON Bernard, Intelligence économique, compétitivité et cohésion sociale, juin 2003, p. 27
} 
main invisible du marché (qui semble nier toute action du réseau), ou la théorie de l'agence (qui porte son regard essentiellement sur les relations entre les actionnaires et le dirigeant de l'entreprise). Nous nous proposons maintenant (a) de passer en revue trois approches de la coordination inter firmes qui renvoient à des types de réseaux particuliers, et donc à des questions d'intelligence économique différentes. Nous nous efforcerons ensuite (b) de voir ce que peut avoir de spécifique le management de ces entreprises qui se coordonnent en réseau.

\section{a. Réseaux et modes de coordination économique.}

\section{$\checkmark$ La coordination bureaucratique et les réseaux prescrits}

La coordination inter firmes est dite " bureaucratique » ou " tutélaire » lorsqu'elle est mise en œuvre par des acteurs hors marché [Etats, organisations internationales, Communauté Européenne...] qui imposent ou recommandent un mode de coordination des activités économiques par la loi, la décision administrative ou judiciaire. Planification impérative ou indicative autrefois, nationalisations puis dénationalisations, réglementation des concentrations et dispositifs anti-trusts, dispositions du droit de la concurrence... imposent ou orientent la coordination d'acteurs économiques dont la puissance publique doute qu'ils prendront, spontanément, les décisions les plus pertinentes pour le système économique dans son ensemble. Les analyses des chercheurs en ce domaine portent sur le caractère purement bureaucratique ou plutôt interventionniste des procédés retenus, sur l'évaluation de la performance obtenue, sur les modes de fonctionnement des acteurs hors marché...

Lorsque le député Carayon demanda en 2003 la mise en place de Comités de Pilotage Régional d'Intelligence économique (CPRIE), d'Agences Régionales d'Intelligence Economique (ARIE), de Pôles de compétitivité etc, il se situait dans cette logique interventionniste. II en va de même lorsqu'il stipule que " le fonctionnement des réseaux essentiels et la sécurité des infrastructures vitales doit relever de l'instance politicoadministrative définie par le gouvernement, en s'appuyant sur le Secrétariat Général de la Défense Nationale (SGDN), les Hauts Fonctionnaires de Défense (HFD) et en particulier celui du ministère de l'Economie, des Finances et de l'Industrie ${ }^{23}$. "

Dans ce schéma de lecture, les réseaux rencontrés sont d'abord des réseaux institutionnalisés, missionnés, lesquels doivent mailler un dispositif impliquant obligatoirement les différentes strates de l'Etat et les collectivités territoriales et, autant que faire se peut, les acteurs privés du marché de l'intelligence économique et les entreprises ayant besoin du dispositif. Nous devrons intégrer ces réseaux là dans notre approche méthodologique en tant que réseaux prescrits. Ils ont leur propre mode de management dans lequel la position hiérarchique des membres des différentes institutions est essentielle. Il est de bonne méthode que de ne pas désigner pour participer à ce genre de réseau des personnes de rang inférieur.

$\checkmark$ Coordination hiérarchique et réseaux d'entreprises.

L'expression coordination hiérarchique est employée pour désigner les opérations de rachat total ou partiel d'entreprises conduisant à une intégration économique horizontale (concentration du secteur) ou verticale (maîtrise d'une filière amont/aval de production/distribution). Les entreprises absorbées, sous une forme juridique ou une autre, passent sous le contrôle hiérarchique de l'entreprise dominante qui coordonne l'activité de l'entité dans son ensemble. L'explication de ces comportements stratégiques réside, selon les chercheurs, dans la recherche d'économies sur les coûts de transaction générés par la

\footnotetext{
${ }^{23}$ Idem, p. 43
} 
coordination sur le marché $\left[\mathrm{COASE}^{24}\right.$, WILLIAMSON $^{25}$ ] : gérer la relation avec le fournisseur que l'on a acquis coûte moins cher que de négocier constamment avec lui, par exemple.

Les formes usuelles de la coordination hiérarchique sont l'absorption, la fusion, la filialisation... Elles conduisent à l'émergence de réseaux d'entreprises stratégiques qui ont fait l'objet de multiples analyses sur l'objet des accords de coordination, leur importance pour les firmes, la place de la firme pivot ${ }^{26}$, les types d'alliances ${ }^{27}$ et leurs configurations ${ }^{28}$, leurs formes juridiques, les profils des initiateurs des coopérations inter firmes, leurs objectifs, leur degré de coopération, le caractère transitoire ou non de ces coopérations ${ }^{29} \ldots$

Les réseaux d'entreprises qui participent de cette coordination sont une part essentielle de la force de frappe économique d'un pays. Ils intéressent donc l'intelligence économique à plusieurs titres:

- veille stratégique sur les principaux réseaux concurrents,

- recherche du maintien dans le giron économique national des réseaux natifs par la protection contre des offensives sur leur capital ${ }^{30}$,

- assistance dans la lutte contre les info-agressions ou l'espionnage économique...

Dans le domaine de l'innovation tout spécialement, les exemples ne manquent pas d'acquisitions d'entreprises destinées à capter les capacités de recherche. L'affaire Gemplus a marqué les esprits ${ }^{31}$; elle n'est pas la seule.

$\checkmark$ Coordination léonine et entreprises de réseaux.

Nous regroupons sous l'appellation personnelle de " coordination léonine " les situations de marché dans lesquelles, par sa seule puissance économique, un acteur du marché, ou un tout petit nombre d'acteurs, est en mesure s'imposer sa volonté stratégique ou d'orienter fortement le fonctionnement du marché. Ces configurations sont bien connues : monopole, monopsone, oligopole, oligopsone, concurrence monopolistique... Elles font l'objet d'une attention forte en Europe de la part d'une Commission qui considère qu'elles sont des entraves à la concurrence et, ce faisant, qu'elles captent une partie du surplus économique auquel pourraient prétendre les consommateurs. Ce que les économistes ont démontré depuis longtemps.

Les entreprises concernées sont très fréquemment des entreprises de réseau (électricité, télécommunications, gaz, circulation ferroviaire, banques, distribution postale, grande distribution alimentaire...). L'intelligence économique développée par ces entreprises est

\footnotetext{
${ }^{24}$ COASE R.H. , «La nature de la firme », Revue Française d'Economie, 3 vol. II, hiver 1987, p. 133 à 160. Traduction de : "The nature of the firm », Economica, novembre 1937, p. 386 à 405

${ }^{25}$ WILLIAMSON Oliver E. The Economic Institutions of Capitalism. Firms, Markets, Relational Contracting, 1985Free Press.

${ }^{26}$ LECOQ Xavier, Le pivot: figure emblématique des réseaux d'entreprises, Les Cahiers de la Recherche, Centre Lillois d'Analyse et de Recherche sur l'Evolution des Entreprises, novembre 1999

${ }^{27}$ BRESSAND Albert \& DISTLER Catherine, La planète relationnelle, Editions Odile Jacob, Paris, $1995,290 p$

${ }^{28}$ GARETTE Bernard et DUSSAUGE Pierre, Les stratégies d'alliance, Editions d'Organisation, 1995, $277 \mathrm{p}$

${ }_{29}$ GENIAUX Isabelle \& MIRA-BONNARDEL Sylvie, Le réseau d'entreprise : aboutissement d'une trajectoire organisationnelle ou forme transitoire d'organisation, Xlle conférence de l'Association Internationale de Management Stratégique, juin 2003

${ }^{30} \mathrm{ON}$ se souvient des cafouillages autour du rachat d'Arcelor par Mittal Steel

${ }^{31}$ MOINET Nicolas, Les batailles secrètes de la science et de la technologie, Editions Lavauzelle, 2003
} 
indiscutablement une intelligence économique en réseau ${ }^{32}$ en raison du quadrillage territorial qu'elles réalisent. Elle demande une attention particulière des autorités dans un esprit de patriotisme économique.

\section{b. Repères managériaux des acteurs de terrain.}

Réseaux prescrits, réseaux d'entreprises, entreprises de réseaux : chacun s'accorde à dire désormais que le réseau est un mode d'organisation opératoire et qu'il ne saurait se manager comme se conduit un ensemble hiérarchisé ${ }^{33}$. Pour autant, se départir des pratiques acquises dans l'entreprise traditionnelle confronte à la difficulté de rechercher d'autres variables managériales. Nous en répertorions ici cinq, mis en avant par les acteurs de terrain, qui conduisent à considérer que le réseau et l'entreprise méritent des approches distinctes.

$\checkmark$ L'effet de milieu, reconnu par exemple par une étude menée en Alsace ${ }^{34}$ sur réseaux innovateurs, un effet de milieu caractérisé par des « relations technologiques parallèles aux échanges avec les clients et les fournisseurs ainsi que des relations informelles ${ }^{35}$. Nathalie Dubost a montré l'importance de ces relations de réseau en tant que réponse stratégique des moyennes entreprises face à leur dépendance économique: " plus précisément, les moyennes entreprises peuvent rendre leurs plus gros clients dépendant d'elles-mêmes en développant un échange basé sur un souci de continuité et s'inscrivant dans une perspective à long terme. » La démarche de l'entreprise doit témoigner " de sa volonté d'accompagner le développement de son client, et donc de maintenir des relations durables avec lui. De même, en allouant des ressources pour le client (formation de vendeurs pour les besoins spécifiques du client, temps passé avec le client, recherche d'informations), l'entreprise démontre qu'elle conçoit cette relation comme un investissement à long terme et qu'elle s'engage pour des relations futures. À cet égard, l'ensemble de ces actions n'est pas dénué de risques pour l'entreprise : en effet, même si le client peut trouver avantage à ce type d'échanges, rien ne garantit à l'entreprise que ces investissements porteront leurs fruits à long terme ${ }^{36} »$.

$\checkmark$ Le caractère plus ou moins formalisé des relations partenariales. La même étude consacrée aux milieux innovateurs semblait percevoir un seuil dans la formalisation des relations de réseaux à partir de 300 employés concernés. Mais beaucoup d'auteurs mettent en avant la part importante du non formel et/ou du non professionnel dans la création des liens qui seront sources de relations d'affaires à venir. Ainsi lisait-on dans l'Usine Nouvelle à propos des associations ou clubs de chefs d'entreprises: "les industriels apprécient leur côté informel, détendu, et même ludique. Par exemple à l'association de matchs de basket, de rugby, ou de concert. Une ambiance qui facilite les contacts, et donc les affaires : on

\footnotetext{
${ }^{32}$ Pour des actions d'influence, par exemple. "Les leçons de la déroute d'EDF à Bruxelles ", Intelligence On Line, $n^{\circ} 440,7$ novembre 2002

${ }^{33}$ SERIEYX Hervé, AZOULAY Bernard, "Mettez du réseau dans vos pyramides », Editions Village Mondial, 1996

${ }^{34}$ HERAUD Jean-Alain \& LAVAL Philippe, "Les réseaux d'innovation des entreprises en Alsace ", Annales des Mines, juillet 1994, pp.48-51

${ }^{35}$ Les réseaux d'innovations territoriaux « peuvent être définis comme des ensembles coordonnés acteurs hétérogènes (Laboratoires privés ou publics, entreprises, clients, fournisseurs, organismes financiers...), géographiquement proches, qui participent activement et collectivement à la conception, à l'élaboration, la fabrication et à la diffusion d'une innovation » (p.4) LOILIER Thomas et TELLIER Albéric, "Structure, fonctionnement et performance des réseaux territoriaux d'innovation : bilan et perspectives de recherche ", octobre 2005, Ecole de management de Normandie, 34 pages

36 DUBOST Nathalie, " un exemple de stratégie de renforcement des moyennes entreprises : l'échange relationnel comme moyen de gérer la dépendance économique envers les clients » Revue Internationale PME, volume neuf, numéro deux, 1996, p. 137
} 
recueille des informations [...] On rencontre de futurs clients ou fournisseurs ${ }^{37}$. " Nous sommes là assez loin du vécu interne à l'entreprise, même si celle-ci s'efforce parfois de mettre en œuvre des actions de gestion des ressources humaines qui empruntent des détours comparables.

$\checkmark$ L'intervention ou non des institutions au premier rang desquelles se trouvent les collectivités locales dans le cadre de leurs politiques de développement territoriales, mais aussi les réseaux développés au niveau national, tels l'ADIT, les ARIST, les CCI...La chambre de commerce et d'industrie de Nîmes a ainsi produit un guide intitulé : "Créer et animer des clubs ou groupements d'entreprises. Outils, méthodes et conseil. " Ce guide met l'accent sur les étapes conduisant à la création du club, sur les bonnes pratiques permettant une animation performante. II y a là, sans doute une démarche méthodologique visant à améliorer la performance des entreprises dans une logique qui relève de l'intelligence économique ${ }^{38}$.

$\checkmark$ La place tenue par les réseaux électroniques dans le fonctionnement des réseaux ${ }^{39}$ et l'action individuelle en réseau. Dans un article paru dans le Competitive Intelligence Magazine de juillet-août 2006, Steve Duncan $^{40}$ analysait par exemple les plateformes électroniques de social networking comme de véritables outils d'intelligence compétitive s'agissant :

«1. d'identifier des experts, spécialement des experts techniques, et en particulier lorsque vous recherchez en dehors de votre industrie et qu'il est possible que la personne n'appartienne pas aux associations habituelles. "

«2. de trouver des employés actuels et d'anciens employés d'entreprises cibles. Ils sont souvent d'excellentes sources d'information. Par exemple, Linkedln propose un service qui vous permet de trouver une personne qui a travaillé pour une entreprise cible et les personnes qui ont travaillé avec celle-ci. »

« 3 . de rassembler des informations masquées, dans la mesure où souvent des individus mettent d'intéressantes données dans leur profil personnel. Dans de tels enregistrements, j'ai souvent trouvé des données relatives aux ventes et de l'information sur la taille et l'organisation des départements et des bureaux d'entreprises. »

$\checkmark$ La dimension culturelle, en particulier la capacité à se repérer dans un système en coopétition. On lisait ainsi dans le magazine L'entreprise en janvier 2000 : « de plus en plus de chefs d'entreprise réalisent que, pour se maintenir face à ouverture des frontières, ils doivent regrouper leurs efforts, ne plus se considérer seulement comme des concurrents, mais aussi comme des partenaires. L'arrivée des nouvelles générations a également contribué à l'évolution des esprits. Les jeunes qui prennent la relève voyagent, parlent l'anglais, utilisent Internet, bref disposent d'une ouverture d'esprit qui favorise la coopération ${ }^{41}$. "

\footnotetext{
${ }^{37}$ LEMARCHAND Jean-Louis, « les réseaux d'influence de l'industrie » l'usine nouvelle, numéro 2541 , 21 mars 1996, page 58

38 Parmi les étapes incontournables recensées : La fixation des objectifs du groupement d'entreprises; la détermination de la cible et des conditions d'adhésion; le choix de la zone géographique ; l'élaboration du plan d'action prévisionnel ; le choix de la structure juridique des règles de fonctionnement correspondantes ; l'interrogation sur les partenaires potentiels; la mise en place d'un plan de financement pour le groupement.

${ }^{39}$ MARCON Christian, "Rencontres de Nîmes : le réseau sous toutes les coutures ", Compte-rendu du Colloque "Les PME dans la nouvelle économie », (Nîmes, 26-27 avril 2000), Veille Magazine, n 35 , juin 2000, pp. 28-29

40 DUNCAN Steve, "Social Networking Systems as Competitive Intelligence Tools », compétitive intelligence magazine, volume neuf numéro quatre, juillet août 2006, pp. 16-19

${ }^{41}$ ARNAUD Rémy, « voisins et concurrents, ils travaillent ensemble » l'entreprise numéro 172, janvier 2000, page 57
} 
En somme, sociologues, économistes, praticiens donnent à qui veut bien les lire des pièces de puzzle qui permettent d'approcher le management de réseaux dans une logique d'intelligence économique. Encore faut-il essayer de mettre les pièces en place et de proposer une approche globale, prélude à un ensemble de travaux plus ciblés. Notre seconde partie s'y emploie.

\section{Méthodologie pour une intelligence économique en réseau.}

Nous nous proposons de présenter maintenant une démarche méthodologique globale en vue de l'intégration de la stratégie-réseau dans un dispositif d'intelligence économique d'entreprise.

Par stratégie-réseau nous entendons la stratégie qui « consiste à créer ou, le plus souvent, à activer et orienter les liens tissés entre des acteurs dans le cadre d'un projet plus ou moins défini. La stratégie-réseau peut en effet aussi bien se situer en amont du projet qu'en aval ${ }^{42}$ " [...] La stratégie-réseau " conduit à mettre en œuvre un dispositif intelligent, c'est-à-dire un système dont on attend en règle générale qu'il scrute l'environnement (veille, vigilance), coordonne les acteurs au service du projet (logique d'interaction) en les faisant profiter de la dynamique d'apprentissage permise par des liens souples. ${ }^{43}$ »

L'intégration [dispositif d'intelligence économique - stratégie-réseau] sera réalisée en deux temps. Nous élaborerons d'abord par étapes successives un schéma d'ensemble articulant une démarche type d'intelligence économique de l'entreprise (présentée sous la forme classique du cycle du renseignement) avec les différents supports réseaux qui, à chaque phase, enrichissent cette démarche. A ce stade, nous irons rechercher dans la première partie des supports de réflexion. Puis, nous suggérerons sept préconisations pour une intelligence économique en réseau.

\subsection{Organisation de l’intelligence économique et stratégie-réseau.}

Nous proposons d'ouvrir notre proposition méthodologique en prenant pour base le cycle du renseignement. Sa simplicité, sa notoriété en font une parfaite introduction à la démarche, même s'il ne résume pas à lui seul, naturellement, la démarche d'intelligence économique. Dans sa formulation de base, le cycle du renseignement (ou cycle de l'information, ou cycle de l'intelligence économique selon les auteurs) prend cette forme.

Figure 1 Le cycle du renseignement

\footnotetext{
${ }^{42}$ MARCON Christian et MOINET Nicolas, « La stratégie-réseau », Edition ZéroHeure, 2000, p. 21

${ }^{43}$ Idem p. 22
} 


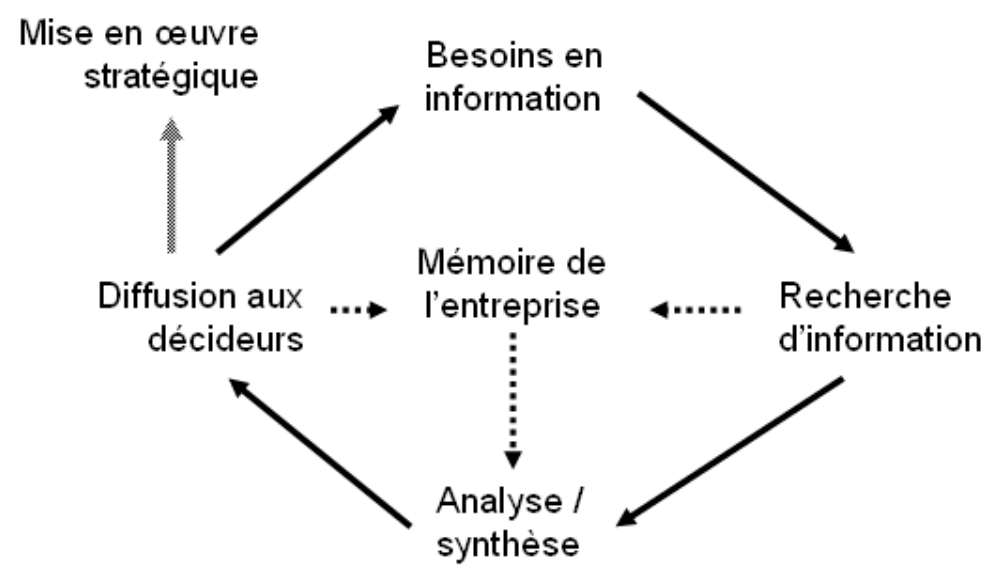

On y lit les phases d'un processus d'intelligence économique simple conduisant à une mise en œuvre stratégique.

Notre première recommandation méthodologique consiste à rapprocher ce cycle d'un autre cycle, celui de la mise en place d'une stratégie-réseau ${ }^{44}$.

Figure 2 Le cycle de la stratégie-réseau

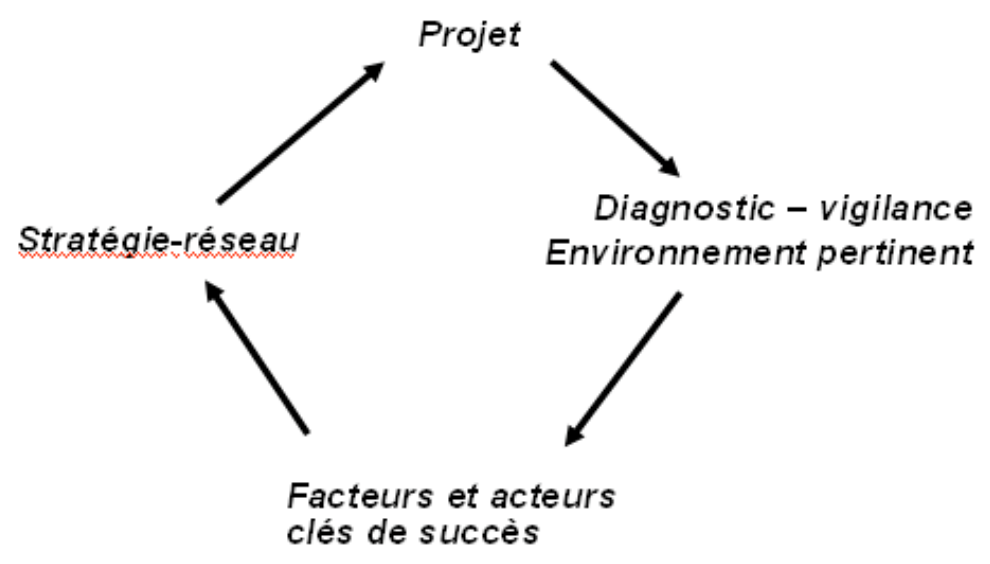

Ce cycle simplifié fait apparaître les quatre temps d'une stratégie-réseau.

Premier temps : la formulation d'un projet. II n'est pas de stratégie-réseau envisageable en dehors d'une idée, d'un projet, d'une ambition. La stratégie-réseau est consubstantielle à l'existence d'un projet. Dans le cas de l'intelligence économique, le projet est celui de l'entreprise: développement interne, rachat d'entreprise, réponse à l'action offensive de déstabilisation par la concurrence, menace... II pourrait être, d'un autre point de vue, celui d'un territoire.

\footnotetext{
Une première formulation de ce cycle, plus détaillée et différente dans sa forme, avait été proposée lors du colloque VSST 2006, MARCON Christian \& MOINET Nicolas, "Méthodologie pour un renforcement du maillon faible », VSST Lille, p. 5
} 
Deuxième temps: diagnostic et surveillance vigilante de l'environnement pertinent de l'entreprise, autrement dit de l'ensemble des domaines et événement susceptibles d'avoir un impact décisif sur le succès du projet ${ }^{45}$. Cet environnement est endogène à l'entreprise.

Troisième temps: à partir du diagnostic réalisé, détermination des facteurs, acteurs et réseaux clés de succès. $A$ un niveau individuel d'analyse, nous résumons ces acteurs clés à une "D.R.E.A.M. team ${ }^{46}$ ": Décideurs, Relais, Eveillés, Ascendants, Mentors. Au niveau d'analyse de l'entreprise, il conviendra d'élargir le panel des réseaux et acteurs clés.

Quatrième temps : mise en œuvre de la stratégie-réseau, qui s'accompagne d'une série de questionnements : existe-t-il un réseau pertinent, ad hoc à la résolution de notre problème ? Faut-il le créer? Les facteurs de succès managérial sont-ils réunis ? Etc.

Le rapprochement des deux schémas montre l'association possible des deux démarches.

Figure 3. Cycle du renseignement et stratégie-réseau (Marcon, 2007)

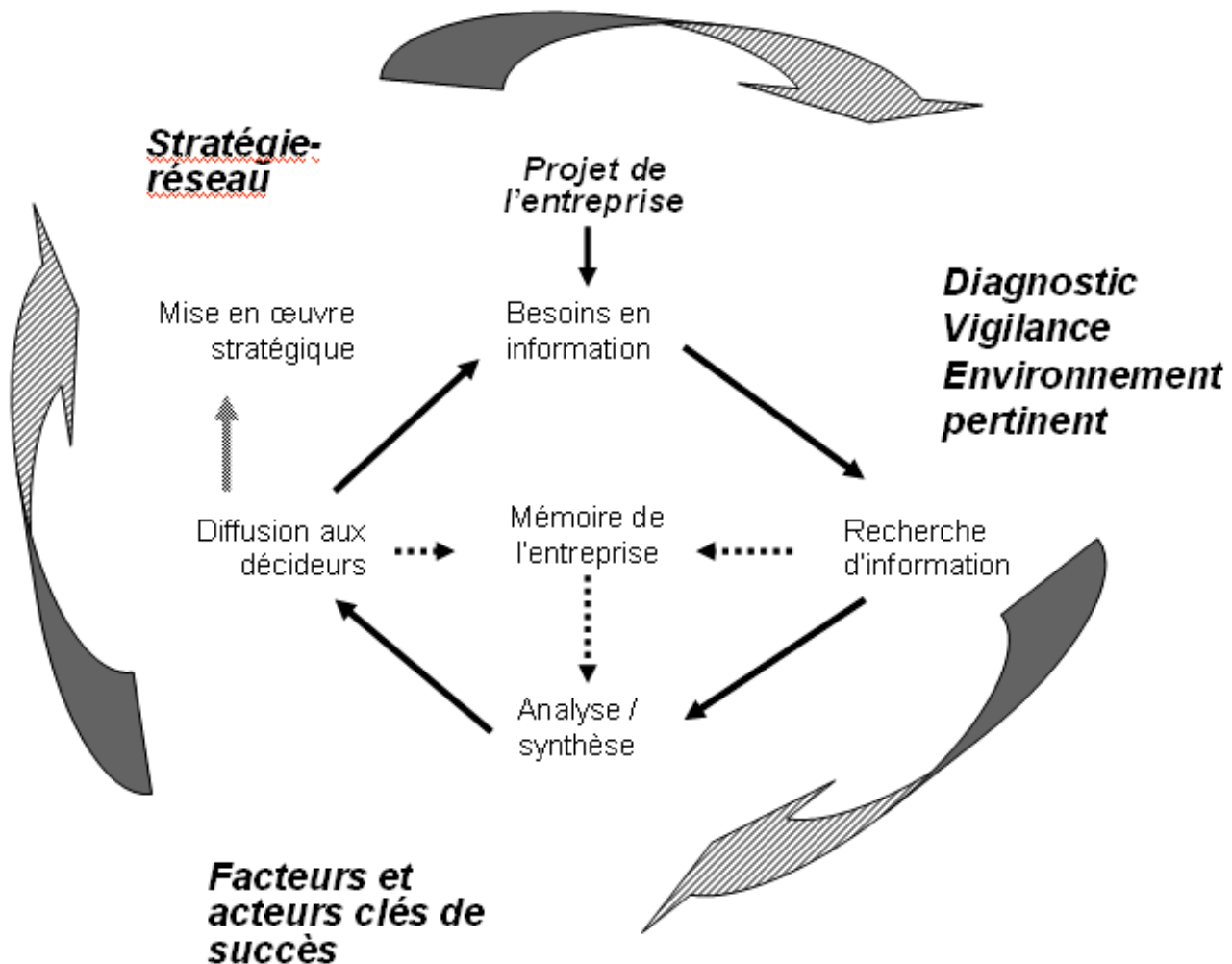

On voit apparaître ici la cohérence et la concordance des démarches. Les besoins en informations découlent naturellement du projet de l'entreprise, mais ils sont aussi déterminés par l'entreprise en considération de son environnement pertinent. Celui-ci, convenablement analysé, soumis à une vigilance permanente, fait émerger des sujets d'observation et génère des recherches d'information. L'analyse des informations captées, entre autres par les réseaux sollicités, conduit à mettre en évidence des facteurs, acteurs et réseaux clés de succès dans l'aboutissement du projet de l'entreprise. Ces informations stratégiques sont

\footnotetext{
45 MARCON Christian, Intelligence économique: l'environnement pertinent comme variable stratégique, thèse de doctorat, 1998.

46 MARCON Christian et MOINET Nicolas, « Développez et activez vos réseaux relationnels », Editions Dunod, $2^{\mathrm{e}}$ édition, avril 2007, p. 136
} 
diffusées aux décideurs qui mettent en œuvre une stratégie réseau. L'ensemble du processus doit être mémorisé afin d'être capitalisé et amélioré.

Partant de ce schéma simple, nous pouvons enfin établir un schéma général qui articule le cycle du renseignement et les phases de la stratégie-réseau.

Figure 4. Schéma d'une intelligence économique d'entreprise en réseau (Marcon, 2007)

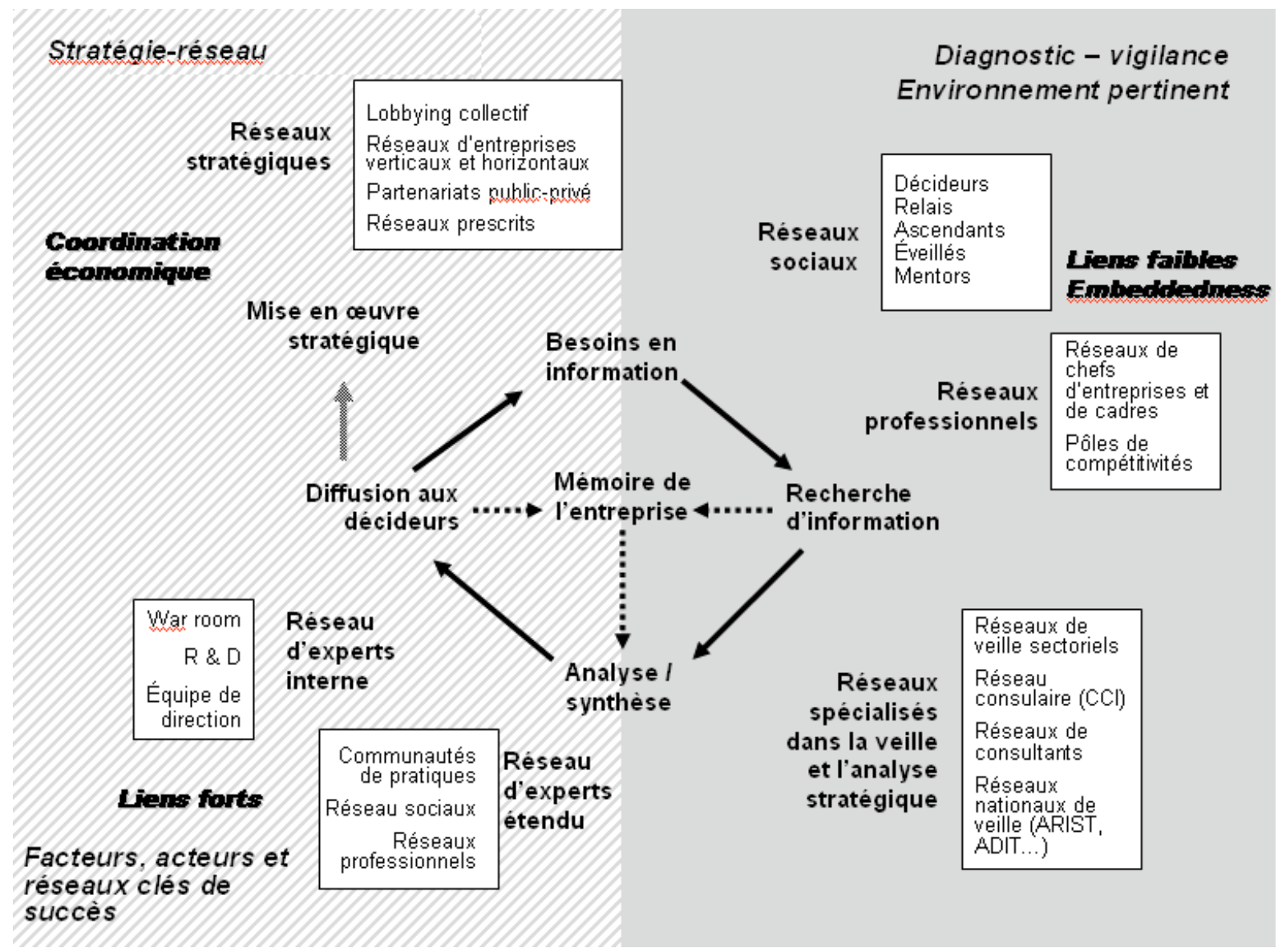

A chaque niveau, certains types de réseaux paraissent devoir être prioritairement sollicités.

$\checkmark \mathrm{Au}$ stade du diagnostic et de la vigilance sur l'environnement pertinent, les réseaux sociaux, les réseaux professionnels, les réseaux spécialisés dans la veille et l'intelligence économique doivent être activés. II apparaît ici que ce sont les réseaux reposant sur des liens faibles, évoqués ci-dessus, qui sont les plus utiles en raison précisément de leur capacité à élargir le champ de vision. Le mécanisme d'embeddedness, également évoqué en première partie, permet la remontée d'informations utiles à l'entreprise : chacun s'efforce de solliciter, dans son réseau social ou professionnel, les individus encastrés dans un milieu qui peut produire des informations utiles à l'observation de l'environnement pertinent.

$\checkmark$ Au stade de la détermination des facteurs, acteurs et réseaux clés de succès : le réseau d'experts internes et le réseau d'experts étendu au-delà des frontières de l'entreprises sont les plus pertinents. Sans doute les liens forts sont-ils les plus souvent sollicités car il s'agit de faire preuve de cohésion dans l'action et de pertinence dans l'interprétation, ce qui implique un fort échange d'information, une grande confiance, une véritable expertise thématique. 
$\checkmark$ Enfin, au stade de la stratégie-réseau, l'entreprise active des réseaux stratégiques au sens où ils accompagnent l'entreprise dans l'action qui est au cœur de son projet. C'est là que trouvent leur pertinence les réflexions en terme de coordination stratégique conduites par les économistes et les spécialistes du management.

\subsection{Recommandations méthodologiques pour une intelligence économique en réseau.}

Pour la mise en œuvre d'une intelligence économique en réseau, il nous semble qu'une méthodologie s'impose, qui rassemble sept recommandations.

\section{Auditer son dispositif réseau.}

Les réseaux de l'entreprise sont souvent des réseaux, sinon de hasard, du moins non construits dans une perspective d'intelligence économique. Fréquemment, le chef d'entreprise adhère à son syndicat professionnel, à un réseau - club service, à un réseau territorialisé, le tout sans avoir conçu son adhésion comme, au-delà de la reconnaissance des pairs et du développement personnel, un engagement stratégique. En fait, il convient, sur la base d'une analyse de son environnement pertinent, de choisir les réseaux qui méritent un investissement stratégique. Ce qui n'est pas incompatible avec une dimension plaisir.

II serait donc cohérent, pour une équipe de direction, d'auditer son dispositif-réseau (qui appartient à quels réseaux ?) et de mettre cette ressource collective en regard des besoins qui émergent de la stratégie de l'entreprise.

2. Sur la base de l'audit précédent, construire son dispositif-réseau afin de trouver une ressource utile à chaque phase du cycle.

II ne saurait être question d'être normatif et de constituer une liste a priori des réseaux pertinents ou non pertinents, fussent-ils labellisés "d'intelligence économique » ou " de compétitivité »! Le choix est directement lié au projet de l'entreprise. II est donc localisé, temporalisé, contextualisé.

La multitude des réseaux a pour conséquence évidente des contributions variables qui doivent être étudiées afin de constituer un dispositif stratégique dans lequel chaque réseau est à tout le moins utile. Nous employons ici les termes "stratégique " et "utile " en référence à la classification de l'information de François Jakobiak, qui distingue l'information fatale, l'information intéressante, l'information utile et l'information stratégique. Cette classification peut aisément être transposée :

$\checkmark$ Information fatale / réseau fatal : l'information fatale arrive forcément dans votre boite aux lettres. Elle vous sera fatale si vous ne savez pas la distinguer rapidement et vous en décharger. Le réseau fatal occupe votre temps pour rien. II vous a fatalement été proposé d'y adhérer.

$\checkmark$ Information intéressante / réseau intéressant. L'information intéressante, comme le réseau intéressant, répondent à une curiosité personnelle. II est normal de s'y intéresser sur son temps libre, mais dommage de s'y consacrer dans un contexte professionnel.

$\checkmark$ Information utile / réseau utile : l'information est dite utile lorsqu'elle concourt à la prise de décision professionnelle. Le réseau utile remplit la même fonction.

$\checkmark$ L'information stratégique / le réseau stratégique : tous deux sont indispensables pour prendre des décisions qui engagent l'avenir de l'entreprise et mettre en œuvre ces décisions. 
Dans tous les cas, des réseaux de liens faibles seuls (réseaux faiblement maillés, avec une activité qui reste relativement peu impliquante) ou de liens forts seuls (réseaux ayant un long vécu en commun, chargé de moments intenses) semblent insuffisants. L'américain Ronald Burt a montré dans le contexte d'individus en compétition au sein d'une entreprise que l'excès de liens forts avec les autres nuit à l'autonomie et constitue une entrave à la progression. Cela nous incite à la vigilance quant à l'excès d'implication du chef d'entreprise dans des réseaux reposant sur des liens très forts.

\section{Comprendre les mécanismes de comportement des réseaux.}

II n'existe pas de modèle de comportement universel des réseaux. Selon les types de réseaux, la manière de se comporter, de solliciter le réseau, d'y contribuer diffère. Ainsi, considérons la matrice suivante ${ }^{47}$.

Figure 5. La matrice dimension structurelle / ressort relationnel (Marcon, 2006)

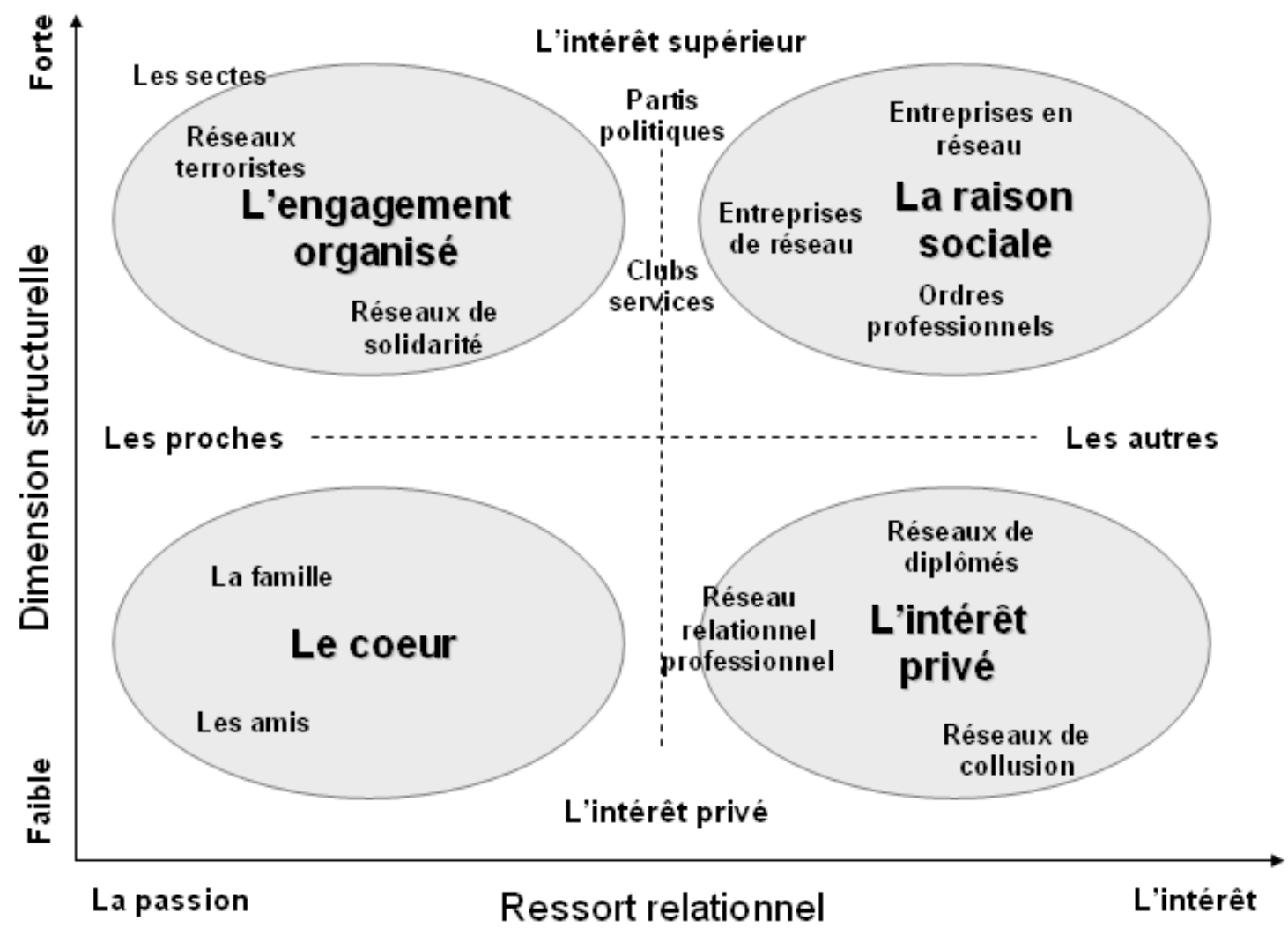

Cette matrice montre la complexité d'approcher les mécanismes de fonctionnement du réseau, selon que ceux-ci fonctionnent sans structure véritable, avec une structure légère ou, au contraire, avec une organisation fortement charpentée. De même, selon que la passion partagée ou l'intérêt commun est le ressort fondamental de rapprochement, les comportements ne seront pas identiques. Pour autant, une grille de lecture simple permet d'aborder le mécanisme du réseau et les comportements observés.

47 MARCON Christian, "Réseaux du personnel et management: la difficile appropriation », Humanisme et entreprise, $\mathrm{n}^{\circ}$ spécial "Les relations interpersonnelles dans les organisations: problème ou ressource? », octobre 2006, pp. 120 
Figure 6. Les variables déterminantes du comportement du réseau (Marcon \& Moinet, 2004)

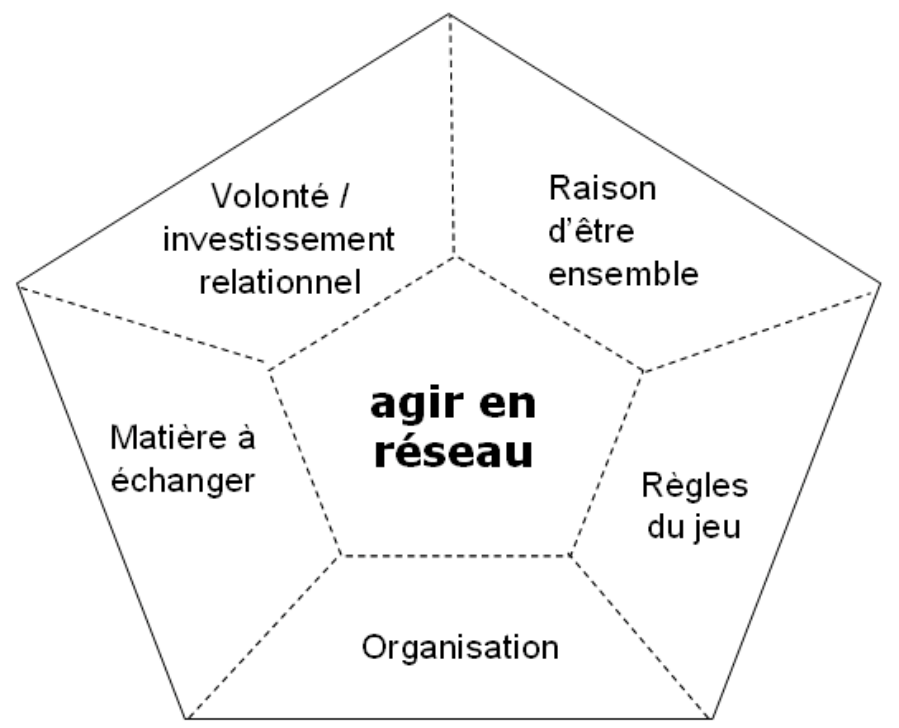

Le degré d'investissement des membres du réseau, tout particulièrement de son/ses animateurs, l'évidence ou du moins la netteté du projet qui rassemble, la clarté des règles comportementales (gouvernance, confiance, gestion du off, intégration et exclusion du réseau...), l'organisation (associative ou non), l'effectivité de la matière à échanger entre les membres (information, soutien, conseils, temps...) sont sans doute les cinq principales clés d'explication de la dynamique comportementale des réseaux ${ }^{48}$ sociaux, des réseaux professionnels, des réseaux spécialisés, réseaux d'experts, etc., ce qui permet d'apprécier leur capacité probable de contribution au projet d'intelligence économique si l'on vient à les solliciter.

II est donc essentiel d'étudier avec méthode sur les points ci-dessus les réseaux cibles avec lesquels l'entreprise envisage de surveiller son environnement, de l'analyser et de conduire ses actions de développement.

\section{Envisager les réseaux comme une ressource qui doit être managée.}

La presse professionnelle de type "grand public » publie plusieurs fois par an des dossiers sur le thème "les meilleurs réseaux pour réussir $"{ }^{49}$. Il se dégage de la lecture de ces articles l'impression qu'il faut, mais aussi qu'il suffit, d'être dans les " bons " réseaux pour bénéficier d'un effet réseau. Une telle approche est évidemment racoleuse et discutable, comme l'a montré le désaccord entre Burt et Lin évoqué dans la première partie de cet article. Etre dans un réseau n'est d'aucune utilité si l'on s'y comporte en pêcheur à la ligne : tout en immobilité et en patience.

Nous avons déjà évoqué la problématique de la centralité : plus l'individu est central, meilleure sera sa capacité profiter des apports du réseau, d'où des stratégies personnelles internes aux réseaux. Au-delà, les réseaux ne peuvent être une ressource que si on les aborde en terme de management. Or tout montre que les talents nécessaires ne sont pas

${ }^{48}$ On consultera avec intérêt les pages consacrées par Guy LEBOTERF à la question du management en réseau. LEBOTERF Guy, « Travailler en réseau. Partager et capitaliser les pratiques professionnelles », Editions d'Organisation, 2004

${ }^{49}$ Dernier en date, « Les réseaux qui marchent en 2007 », L'entreprise, mai 2007, pp. 29-43 
naturellement présents chez chacun. Nous le rappelions dans la première partie de cet article $^{50}$. Même présents, ils doivent être travaillés, comme un sportif s'entraîne. Ce n'est pas un hasard si le site www.placedesreseaux.com propose un guide de quatorze pages intitulé " 10 conseils pour dynamiser votre réseau personnel ». Si l'on considère que le réseau personnel est un instrument au service de l'intelligence économique, il est légitime de chercher là des conseils méthodologiques. Le guide en propose un assez long catalogue, assemblage de suggestions judicieuses davantage que vision d'ensemble.

Les qualités nécessaires au management d'un réseau d'intelligence économique sont-elles substantiellement différentes de celles communément utiles? Sans doute sur certains points : la capacité à créer une confiance forte, donc à gérer le off et la discrétion dans les rapports interpersonnels. Le manageur de réseau doit aussi être diplomate, tenace, proactif, fiable et tenir compte du contexte réseau dans lequel il agit.

Sur la base d'une analyse qui croise le mode de relation choisi (coopératif versus hiérarchique) et le motif principal d'adhésion au réseau (contrat versus connivence), nous avons ainsi mis en exergue quatre talents capitaux pour le management de réseau: le grade, le charisme, la compétence et l'activisme ${ }^{51}$.

Figure 7. Les quatre talents principaux du management en réseau (Marcon \& Moinet, 2004)

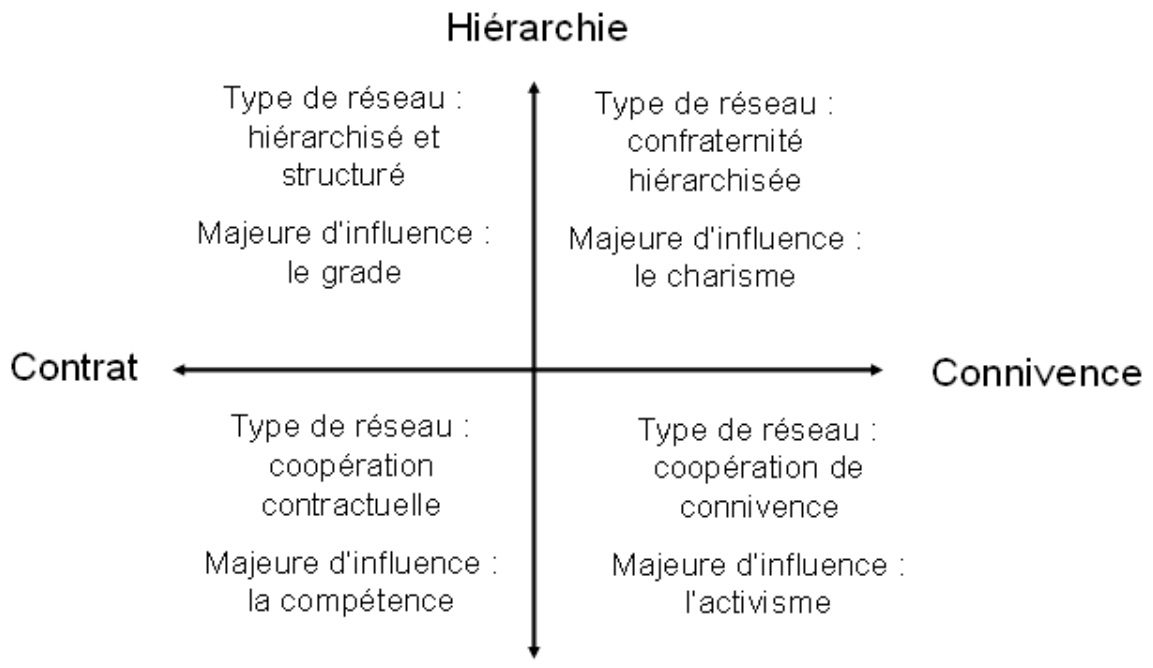

Coopération

\section{Arrêter de fantasmer sur les réseaux et les logiciel dédiés}

En janvier 1996, T.J. Facquer écrivait à propos du réseau: " «Les structures en réseau, mouvantes et imprévisibles, en permanente évolution, et capables de s'adapter rapidement, sont plus efficaces face à des situations complexes que les structures pyramidales rigides. De plus, l'association de plusieurs compétences, relevant de domaines différents, agissant

\footnotetext{
${ }^{50}$ Une étude réalisée sur de futurs diplômés en intelligence économique a montré qu'une proportion non négligeable d'entre eux fait un blocage face à la perspective de développer une démarche en réseau, pourtant directement liée au métier pour lequel ils se forment ! MARCON Christian et MOINET Nicolas, "Enseigner la stratégie-réseau: un défi méthodologique et culturel pour l'intelligence économique ", $1^{\text {er }}$ Colloque Européen d'Intelligence Economique, Poitiers, 27 janvier 2005

51 MASSE Guy, MARCON Christian, MOINET Nicolas, "Les fondements de l'intelligence économique : réseaux et jeux d'influence ", Marketing et Communication. Revue Internationale des Sciences Sociales., $n^{\circ} 3$ - octobre 2006, pp. 84 -103
} 
sur un pied d'égalité, ce qui caractérise le fonctionnement en réseau, doit permettre d'augmenter les capacités d'appréhension de la réalité. Là où un individu, aussi brillant soitil, ne parviendra plus à se représenter une réalité dans toute l'étendue de sa complexité, il pourra, avec l'aide du réseau, trouver la clé qui lui fait défaut. Comme son appartenance à un réseau ne sera pas exclusive de son appartenance à un ou plusieurs autres, il pourra, en fonction de ses besoins, activer tel ou tel réseau, voire ceux de ses collaborateurs. Ainsi, l'appel aux réseaux permet-t-il de démultiplier à l'infini et les possibilités de recours face à des situations a priori indéchiffrables ${ }^{52}$. »

Le discours est excessivement optimiste. Les réseaux ne peuvent pas démultiplier à l'infini les possibilités de traitement de situations complexes, mais ils augmentent les marges d'interprétation du réel et d'action stratégique, sous réserve de les considérer pour ce qu'ils peuvent apporter et non pour le fantasme qu'ils suscitent. Ainsi, on attend beaucoup trop des plateformes de social networking par exemple. L'idée selon laquelle en six rebonds n'importe qui pourrait être connecté à n'importe qui dans le monde, ouvrant ainsi un espace de collaboration infini, est tout à fait de l'ordre du fantasme ${ }^{53}$. Encore faudrait-il que chaque maillon ait une bonne raison de relayer vers le maillon suivant ! De même, il est assez vain d'espérer, grâce au recyclage de logiciels de CRM (Consumer Relationship Management) en logiciels de management de réseaux, cartographier l'ensemble des réseaux des membres du personnel afin d'optimiser leur exploitation au service de l'entreprise ${ }^{54}$. Un bon principe de méthodologie est de considérer l'outil pour ce qu'il est : un bon serviteur. Si certains logiciels et sites web permettent d'enrichir l'analyse réseau, il faut savoir les remettre à leur place :

- www.kartoo.com, en identifiant les occurrences d'apparition conjointes d'individus permet de présumer l'existence de liens entre eux. A condition de se méfier des homonymes. Et à supposer que ces individus soient visibles sur Internet, ce qui n'est pas le cas général.

- www.societe.com permet de savoir qui participe aux mêmes conseils d'administration, donc, là encore, de déceler des réseaux. Si les entreprises n'ont pas préféré payer plutôt que de donner leurs informations.

- www.viadeo.com doit permettre d'identifier des personnes qui se disent intéressées par une même thématique et de préciser leur degré de proximité relationnelle en nombre de rebonds. Encore faut-il qu'elles aient choisi le même mot clé, et qu'elles soient sur viadéo.

- Le logiciel Freemind permet des représentations de réseaux sociaux, de réseaux professionnels, de réseaux d'entreprises... avec la limite d'une représentation centrée sur un cœur de réseau (individu, entreprise) qui ne reflète pas nécessairement la réalité.

- Le logiciel The brain corrige ce défaut en modifiant l'angle de vue du réseau selon l'acteur qui est étudié afin que celui-ci se retrouve central et que l'ensemble soit repositionné par rapport à lui. Mais la manipulation du logiciel n'est pas des plus aisées.

Naturellement, certains logiciels professionnels très coûteux offrent des performances plus grandes. Mais on risque de connaître la même déception que celle qui a suivi l'enthousiasme initial des professionnels de l'intelligence économique à l'égard des logiciels de knowledge management : le logiciel ne remplace pas l'absence de démarche et de management.

\footnotetext{
52 Thierry-James FACQUER, «le réseau : une réponse à la complexité » management France, numéro 95, janvier 1996

${ }^{53}$ KLEINFELD Judith S., "Could it be a big world after all ? The six degrees of separation myth", Forthcoming, Society, 2002. Disponible à l'adresse : http://www.uaf.edu/northern/big world.html

54 MARCON Christian, "Réseaux du personnel et management: la difficile appropriation », Humanisme et entreprise,op. cit.
} 


\section{6. (Re)lire les chercheurs et se former.}

II faut brièvement redire que le recours aux logiques de réseau n'est ni naturel, ni simple, ni nécessairement productif. Agir en réseau n'est naturel que pour celui qui a un caractère extraverti, ce qui n'est pas toujours le cas, comme l'a montré l'analyse structurale. Agir en réseau n'est pas toujours simple ni productif : tous ceux qui ont eu l'expérience de réseaux vieillissants, de réseaux amorphes, de réseaux enthousiastes mais débordés, de réseaux injoignables lorsque l'on a besoin d'eux, etc. connaissent les difficultés de solliciter, d'obtenir une information, une action, un financement, une aide quelconque.

Par conséquent, il est urgent de former les veilleurs, chargés d'intelligence économique et autres animateurs de dispositifs territoriaux à l'action en réseau et, dans ces formations, de ne pas en rester aux discours de l'outil, du «truc » ou de l'ardente obligation. Pour ce faire, pas d'autre solution que de (re)lire : les ouvrages désormais assez nombreux sur ce sujet, les travaux des chercheurs en français lorsqu'ils existent (Revue Française de Gestion, Actes de l'Association Internationale de Management Stratégique par exemple), en anglais plus souvent (Academy of Management Review \& Journal, Administrative Science Quaterly...) et, de plus en plus, sur Internet.

\section{Poser clairement la déontologie de son action en réseau.}

La question de la déontologie est incontournable en intelligence économique d'une manière générale $^{55}$. Le recours aux réseaux ne saurait échapper à cette interrogation. Les affaires d'espionnage ou politico médiatiques ont mis sur la sellette les réseaux qui ont, globalement mauvaise presse, des réseaux facteurs de piston, de désinformation, d'information occulte, d'influence, d'entrave à la concurrence et autres maladies honteuses... Chacun sait que, dans un monde concurrentiel dont les économistes ont montré qu'il vit d'asymétries d'informations créées ou compensées, le recours au réseau est envisagé comme une arme défensive / offensive. De ce fait, l'action en réseau doit être demandée aux acteurs dans le strict respect d'une déontologie.

L'établissement d'une charte de déontologie, parce qu'elle rassure les personnes impliquées dans l'action en réseau quant à l'absence de risque juridique dans le cadre de leur action, est sans doute indispensable pour permettre l'investissement le plus large des acteurs de l'entreprise. Or nous avons besoin en ce domaine de l'action du plus grand nombre, sans se leurrer jusqu'à marteler "Tous réseauteurs!» comme certains se hasardèrent, bien légèrement, à affirmer « Tous veilleurs!"

\section{Conclusion}

Nous avons essayé de montrer que l'action d'intelligence économique en réseau est perfectible grâce à un effort d'information et de formation, ce qui ouvre les portes d'une approche méthodologique du problème.

Effort d'information en acceptant d'entrer dans une littérature non séduisante a priori mais qui rend souvent compte d'expériences scientifiques dont la portée est précise. Dans la première partie de cet article, par exemple, en parcourant les résultats des travaux de l'analyse structurale avec ses interrogations sur les trous structuraux dans les réseaux, sur

\footnotetext{
${ }^{55}$ MARCON Christian \& MOINET Nicolas, «L'intelligence économique », Editions Dunod, collection Topos, 2006, pp. 85-89
} 
les positions individuelles, en distinguant liens forts et liens faibles, en interrogeant les économistes sur les modes de coordination d'entreprises nous avons pu aisément ouvrir des pistes de réflexion, présentées en deuxième partie, sur les réseaux pertinents à chaque phase du cycle de l'action d'intelligence économique.

Effort de formation car, de même que l'entraînement améliore la performance du sportif, la formation aux logiques de réseaux dans leurs dimensions humaine, économique, managériale, déontologique permet d'enrichir le dispositif d'intelligence économique de l'entreprise ou du territoire. De nombreux acteurs en sont convaincus, qui se sont lancés dans de telles formations. Nous citerons, à titre d'exemples, les Réseaux de Développement Technologiques, le Conseil Régional Nord-Pas-de-Calais, le Medef de la Vienne...

Nous ne sommes pas au bout de la réflexion, mais une porte se ferme alors qu'une autre s'ouvre. La porte du discours galvanisateur reposant sur une intime conviction encouragée par un effet de mode se clôt. Non, les réseaux sociaux ne peuvent pas tout. Non, les réseaux électroniques n'ont pas périmé les réseaux humains. Non, toute l'information n'est pas dans les réseaux Internet. Non, le réseau n'est pas naturellement la panacée de l'intelligence économique... La porte qui s'ouvre est celle de la méthode. Matrices, modèles, expérimentations, analyses qualitatives nous permettront de renforcer nos dispositifs, comme la réflexion sur la veille, sur le knowledge management, la sécurité l'ont permis.

A charge pour les praticiens en entreprise, les consultants, les institutionnels de faire un effort d'intelligence collective avec les chercheurs universitaires. C'est bien le moins que l'on puisse attendre des acteurs de l'intelligence économique.

\section{Bibliographie}

ARNAUD Rémy, "voisins et concurrents, ils travaillent ensemble », L'entreprise $n^{\circ} 172$, janvier 2000

BAUMART Philippe, "Stratégie et surveillance des environnements concurrentiels ", Editions Masson, Paris, 1991

BESSON Bernard et POSSIN Jean Claude, «Du renseignement à l'intelligence économique », Editions Dunod, Paris, 1996

BOURDIEU Pierre (1985), The forms of capital, in Richardson J.G. (éd.), Handbook of theory and research for the sociology of education, New-York, Greenwood, p. 241-258.

BRESSAND Albert \& DISTLER Catherine, La planète relationnelle, Editions Odile Jacob, Paris, 1995

BURT Ronald S. (1992), Structural Holes. The Social Structure of Competition, Cambridge (Mass.), Harvard University Press

CARAYON Bernard, Intelligence économique, compétitivité et cohésion sociale, juin 2003

CHOLLET Barthélémy, Le rôle du réseau personnel de l'ingénieur en $R$ \& $D$ : le cas du secteur des micro et nano technologies, thèse de doctorat, décembre 2005

COASE R.H. , "La nature de la firme », Revue Française d'Economie, 3 vol. II, hiver 1987, p. 133 à 160. Traduction de : «The nature of the firm », Economica, novembre 1937, p. 386 à 405

DEBRAY Régis, Introduction à la médiologie, Presses Universitaires de France, Paris, 2000 DUBOST Nathalie, "Un exemple de stratégie de renforcement des moyennes entreprises: l'échange relationnel comme moyen de gérer la dépendance économique envers les clients » Revue Internationale PME, volume neuf, numéro deux, 1996

DUNCAN Steve, "Social Networking Systems as Competitive Intelligence Tools", compétitive intelligence magazine, volume neuf numéro quatre, juillet août 2006, pp. 16-19 
ENSLEY, M.D., PEARSON, A.W., \& AMASON, A.C. (2002). Understanding the Dynamics of New Venture Top Management Teams: Cohesion, Conflict and New Venture Performance, Journal of Business Venturing, 17(4), 365-386.

FACQUER Thierry James, « le réseau : une réponse à la complexité » management France, numéro 95, janvier 1996

GARETTE Bernard et DUSSAUGE Pierre, Les stratégies d'alliance, Editions d'Organisation, 1995

GENIAUX Isabelle \& MIRA-BONNARDEL Sylvie, Le réseau d'entreprise: aboutissement d'une trajectoire organisationnelle ou forme transitoire d'organisation, Xlle conférence de l'Association Internationale de Management Stratégique, juin 2003

GERAUDEL Mickaël, Les retombées du réseau personnel du dirigeant d'entreprise : la personnalité a-t-elle un rôle ? Actes de la $16^{\mathrm{e}}$ Conférence internationale du management stratégique, Montréal, juin 2007

GRANOVETTER Mark, The strengh of weak ties : a network theory revisited, Sociological Theory, volume 1 (1983), p. 201-233

GROSSETTI Michel et BES Marie-Pierre, Les effets de la proximité spatiale dans les relations entre organisations : encastrements et découplages, Communication pour les 3éme journées de la Proximité, 13 et 14 décembre 2001, Centre d'étude des rationalités et des savoirs - Université Toulouse-le-Mirail

HERAUD Jean-Alain \& LAVAL Philippe, «Les réseaux d'innovation des entreprises en Alsace », Annales des Mines, juillet 1994, pp.48-51

JAKOBIAK François, "L'intelligence économique en pratique », Editions d'organisation, Paris, 1998,

KLEINFELD Judith S., " Could it be a big world after all ? The six degrees of separation myth", Forthcoming, Society, 2002. http://www.uaf.edu/northern/big world.html

LEBOTERF Guy, «Travailler en réseau. Partager et capitaliser les pratiques professionnelles », Editions d'Organisation, 2004

LECOQ Xavier, Le pivot : figure emblématique des réseaux d'entreprises, Les Cahiers de la Recherche, Centre Lillois d'Analyse et de Recherche sur l'Evolution des Entreprises, novembre 1999

LEMARCHAND Jean-Louis, " les réseaux d'influence de l'industrie " l'usine nouvelle, numéro 2541, 21 mars 1996

LENGRAND Louis \& CHATRIE Isabelle, «Les réseaux d'entreprise et l'économie de la connaissance. Une étude de terrain mené en Europe et au Canada » une initiative de la direction générale d'entreprise de la commission européenne, novembre 1999

LEVET Jean Louis et al., Présentation du référentiel AFDIE en intelligence économique, Actes des $4^{\mathrm{e}}$ rencontres nationales d'intelligence économique, 8 novembre 2001

LIN Nan, Les ressources sociales: une théorie du capital social, Revue Française de Sociologie, Vol. 36, No. 4, Analyses de réseaux et structures relationnelles (Oct. - Dec., 1995), pp. 685-704

LOILIER Thomas et TELLIER Albéric, "Structure, fonctionnement et performance des réseaux territoriaux d'innovation : bilan et perspectives de recherche », octobre 2005, Ecole de management de Normandie,

MARCON Christian, Intelligence économique : l'environnement pertinent comme variable stratégique, thèse de doctorat, 1998.

MARCON Christian, MOINET Nicolas, « La stratégie-réseau », Edition ZéroHeure, 2000

MARCON Christian, "Rencontres de Nîmes : le réseau sous toutes les coutures ", Compterendu du Colloque "Les PME dans la nouvelle économie », (Nîmes, 26-27 avril 2000), Veille Magazine, $n^{\circ} 35$, juin 2000

MARCON Christian et MOINET Nicolas, «Enseigner la stratégie-réseau: un défi méthodologique et culturel pour l'intelligence économique », $1^{\mathrm{er}}$ Colloque Européen d'Intelligence Economique, Poitiers, 27 janvier 2005

MARCON Christian \& MOINET Nicolas, "L'intelligence économique », Editions Dunod, collection Topos, 2006 
MARCON Christian \& MOINET Nicolas, « Méthodologie pour un renforcement du maillon faible », VSST Lille, 2006

MARCON Christian, "Réseaux du personnel et management: la difficile appropriation », Humanisme et entreprise, $\mathrm{n}^{\circ}$ spécial "Les relations interpersonnelles dans les organisations : problème ou ressource ? », octobre 2006

MARCON Christian et MOINET Nicolas, « Développez et activez vos réseaux relationnels », Editions Dunod, $2^{\mathrm{e}}$ édition, avril 2007

MARTINET Bruno, RIBAULT Jean-Michel, «La veille technologique concurrentielle et commerciale », Les éditions d'organisation, Paris, 1989

MASSE Guy, MARCON Christian, MOINET Nicolas, "Les fondements de l'intelligence économique: réseaux et jeux d'influence ", Marketing et Communication. Revue Internationale des Sciences Sociales., $\mathrm{n}^{\circ} 3$ - octobre 2006, pp. 84 -103

MERCKLE Pierre, "Les réseaux sociaux. Les origines de l'analyse des réseaux sociaux », CNED-ENS/Ish 2003-2004

MOINET Nicolas, Les batailles secrètes de la science et de la technologie, Editions Lavauzelle, 2003

PICHOT-DUCLOS Jean, "L'intelligence économique : pour un modèle français ", Défense Nationale, janvier 1994, p. 73 à 85

PINELLI Nathalie, Corse, l'île réseau(x), mémoire pour la validation d'un master Intelligence Economique et Communication Stratégique - Université de Poitiers 2007.

SERIEYX Hervé, AZOULAY Bernard, "Mettez du réseau dans vos pyramides », Editions Village Mondial, 1996

UCBASARAN, LOCKETT, WRIGHT \& WHESTHEAD (2003), Entrepreneurial Funders Teams: Factors Associated with Entry and Exit, Entrepreneurship Theory and Practice, pp. 107-128.

WEINSTEIN Olivier, Modèles d'innovation et modes de coordination inter et intraentreprises, in Palloix C., Rizopoulos Y. (Eds.), Firmes et économie industrielle, Paris, 1997, L'Harmattan

WILLIAMSON Oliver E. The Economic Institutions of Capitalism. Firms, Markets, Relational Contracting, 1985Free Press. 